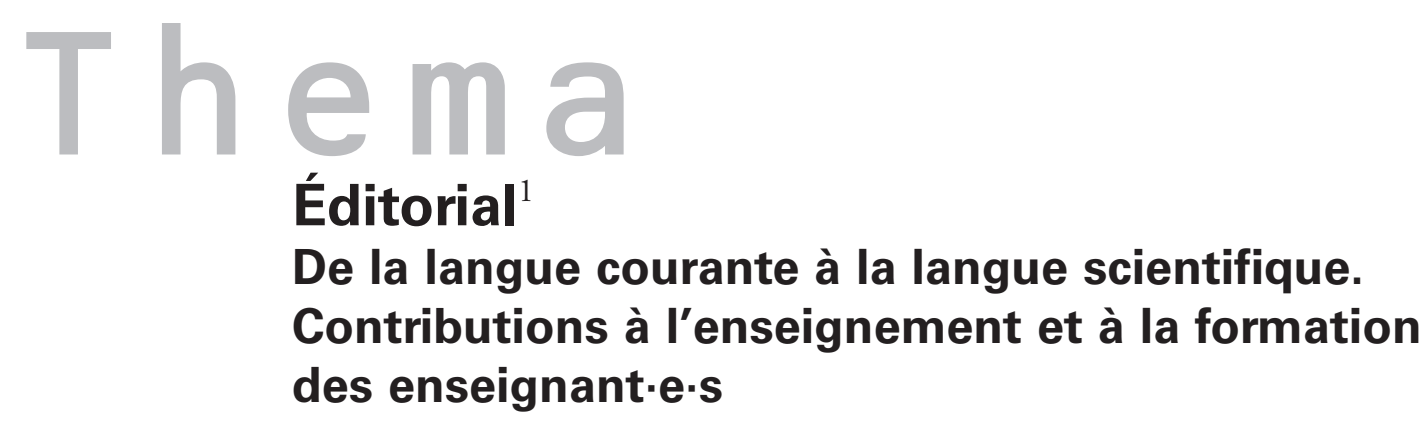

Alain Pache, Haute École Pédagogique Vaud
Patrick Roy, Haute École Pédagogique Fribourg

Quels que soient les sujets abordés à l'école, la question du langage se pose de manière cruciale. Dans l'enseignement de la géographie, par exemple, il n'est pas pareil de parler d'un·e migrant·e, d'un·e réfugié.e, d'un·e immigrant·e, d'un·e émigrant·e ou encore d'un·e sans-papiers. Dans le même ordre d'idée, les outils sémiotiques (Nonnon, 2008) utilisés pour médiatiser le réel ne sont pas interchangeables et il n'est jamais anodin d'en choisir un plutôt qu'un autre. Par ailleurs, comme le titre de ce dossier l'indique, il existe un continuum entre, d'un côté, la langue courante, que l'on pourrait assimiler au sens commun et, de l'autre, la langue scientifique, qui est une sorte d'idéal à atteindre à l'école. En effet, comme l'ont rappelé Moscovici et Hewstone (1984), la science peut se définir comme un " travail d'élucidation et de mise en ordre de matériaux populaires, religieux et même magiques " (p. 542). Cela revient à dire que la science serait une sorte de " sens commun systématisé " (p. 542). C'est dans cette perspective que nous avons peu à peu constitué un recueil de textes qui montrent en quoi un travail explicite sur le langage permet de construire des savoirs utiles pour l'enseignement et la formation aux disciplines scientifiques. Nous avons fait ce choix, car il nous semblait utile de réfléchir à ces questions dans le prolongement d'un symposium qui visait à problématiser les pratiques langagières au sein des démarches d'investigation scientifique en sciences de la nature et en sciences humaines et sociales. ${ }^{2}$ Ce choix s'explique aussi par le fait que, très souvent, la dimension langagière fait figure d'impensé dans ces disciplines, ce qui a pour conséquence qu'elle ne fait que rarement l'objet d'un enseignement explicite (Audigier, 2007).

Ce dossier fait en outre écho aux travaux récents portant sur la verbalisation (Balslev, et al., 2019), sur les interactions didactiques (Bigot, 2016, Filliettaz \& Schubauer-Leoni, 2008), sur les gestes professionnels (Bucheton et Dezutter, 2016), sur le métalangage (Fadhlaoui, 2016) ou encore sur la narration de recherche en mathématiques (El-Mekaoui et Regnier, 2018). Il s'en distingue toutefois en mettant l'accent sur l'enseignement et l'apprentissage des disciplines scientifiques et en mobilisant des cadres théoriques qui font référence dans ce champ.

Tous les "domaines de l'activité humaine, aussi variés soient-ils, se rattachent toujours à l'utilisation du langage " (Bakthine, 1984, p. 265). La communauté scientifique n’échappe pas à cette dimension puisqu'elle est fondamentalement basée sur des pratiques langagières qui lui sont propres et constitutives des savoirs qu'elle produit (Lhoste, 2008). En référence à Popper (1991), Orange (2003) souligne que l'activité scientifique ne se réduit pas à la conduite des démarches scientifiques, mais qu'elle se caractérise par une attitude critique qui ne peut se développer que par des échanges écrits et oraux. Latour et Woolgar (1988) mettent en évidence que c'est grâce aux interactions langagières spécifiques, notamment interpellées au sein des débats scientifiques, que les chercheur.e.s développent leur raisonnement, élaborent leur pensée et produisent le savoir scientifique. Si en aval les productions orales et écrites des savant.e.s ont pour but de rendre compte au monde extérieur des activités qu'elles et ils réalisent, en amont, elles constituent les principaux vecteurs de la construction des savoirs. Cette construction nécessite la mobilisation d'une pluralité de registres de représentation sémiotique comme le rappelle Chevallard (1991) en stipulant que ceux-ci :

émergent d'un système de pratiques où sont manipulés des objets matériels qui se découpent dans différents registres sémiotiques : registre de l'oral, des mots ou expressions prononcés; registre du gestuel ; domaine de la scription, de ce qui est écrit ou dessiné (graphismes, formalismes, calcul, etc.), c’est-à-dire registre de l'écrit (p. 110).

1 La version allemande de cet éditorial a été rédigée avec la collaboration d'Ingo Thonhauser, de la Haute école pédagogique du canton de Vaud (HEP Vaud).

2 Ce symposium a été organisé par le Conseil académique des hautes écoles romandes en charge de la formation des enseignant.e.s et a eu lieu à la HEP Fribourg les 27 et 28 avril 2017. 
À ce sujet, Duval (1996), en citant Vygotski (1985), accorde un statut important au signe dans la formation des concepts scientifiques : "L'élément central (du processus de formation des concepts) est l'utilisation du signe, ou du mot, comme moyen permettant à l'adolescent.e de soumettre à son pouvoir ses propres opérations psychiques, de maitriser le cours des propres processus psychiques » (p. 150). De même, le signe est au cœur de la modélisation en sciences. Cette activité, qui constitue le fonctionnement essentiel des sciences (Bachelard, 1979 ; Bunge, 1973 ; Tiberghien, 1994), permet de construire et réviser de nombreux modèles visant à décrire, expliquer et prédire des phénomènes, lesquels peuvent être représentés par une grande diversité de registres de représentation sémiotique, tels que les objets matériels, les schémas, les représentations algébriques, graphiques ou vectorielles, les simulations informatiques, les analogies, etc. (Bécu-Robinault, 2005; Boulter et Gilbert, 2000 ; Drouin, 1988 ; Gilbert et Boulter, 2000 ; Hasni, 2010 ; Halbwachs, 1974 ; Robardet et Guillaud, 1997 ; Walliser, 1977). C’est pourquoi plusieurs épistémologues définissent les modèles en sciences sur la base des systèmes de signes supportant leur expression. C'est le cas d'Halbwachs (1974) qui dit :

À toute situation physique du "monde réel", il correspondra, dans la "science physique", [...] un (ou plusieurs) systèmes de "signes" consistant en des figures, des graphiques, des symboles mathématiques, ou plus simplement des propositions formées avec des mots, systèmes qui seront alors censés représenter la situation. À un tel système de signes, nous donnerons le nom de modèle et nous poserons la définition : connaître une situation, c'est la représenter par un modèle. (p. 39-40)

Cette référence aux sciences a conduit plusieurs didacticien.ne.s francophones qui ont contribué à élaborer le paradigme socioconstructiviste à s'intéresser aux pratiques langagières à l'école, et plus particulièrement aux relations entre ces pratiques et les apprentissages scientifiques qui s'opèrent au sein des interactions langagières enseignant·e-élèves et élèves-élèves (Fillon et Peterfalvi, 2004 ; Jaubert et Rebière, 2000, 2001 ; Orange, 2003 ; Schneeberger, 2003 ; Schneeberger et Ponce, 2004 ; Schneeberger et al., 2007). En faisant place au débat scientifique, la classe se transforme en une communauté discursive scientifique scolaire, mettant en jeu des processus de négociation comparables à ceux qui fonctionnent dans la communauté des savant.e.s (Johsua et Dupin, 1993 ; Lhoste, 2008 ; Orange, 2003). En référence aux travaux de Bachelard $(1993,1998)$ et de Canguilhem $(1955,1988)$ qui postulent que les savoirs scientifiques sont des savoirs raisonnés, résultants de la construction de problèmes explicatifs, Orange (1997) envisage le débat scientifique en classe comme une situation permettant d'explorer le " champ des possibles » et de construire des raisons, à savoir des contraintes et des nécessités, constitutives des savoirs scientifiques. Dans ce cadre, les élèves sont amené.e.s à formuler des arguments et à en expliciter les fondements, pour construire des explications possibles des phénomènes (Orange, 2003). Dans le monde anglo-saxon, Driver, et al. (1998) et Osborne, et al. (2001) soulignent les apports de l'argumentation pour la compréhension des concepts, le développement des habiletés d'investigation et la construction d'éléments d'épistémologie chez les élèves. Dans le monde germanophone, divers spécialistes ont réfléchi à la question de promouvoir le potentiel de tou-te-s les élèves de façon optimale. Dans ce contexte, elles et ils ont développé le concept de Bildungssprache ou "compétence en littératie " (Becker-Mrotzek, Schramm, Thürmann et Vollmer, 2013 ; Cummins, 1979 ; Feilke, 2012 ; Fürstenau et Gomolla, 2009, 2011, 2012 ; Gogolin, 2010). Pour ces chercheur.e.s, la langue de l'éducation n'est pas la langue de communication de tous les jours, mais bien des procédures linguistiques qui permettent la description, la comparaison, l'explication, l'analyse ou encore l'argumentation. Il s'agit donc de fournir des outils aux élèves, afin qu'elles et ils comprennent le langage scientifique, détaché du contexte de la situation étudiée.

Dans le champ des sciences humaines et sociales, les travaux abordent l'usage des récits pour dire le monde (Audigier, 2000, 2007), les pratiques argumentatives et le débat (Audigier, et al., 2011), les pratiques langagières fondées sur des outils spécifiques - la liste, le tableau - et permettant la problématisation (Doussot, 2011), le rôle du langage dans la construction de compétences éthiques (Lalagüe-Dulac, 2012) ou encore les registres sémiotiques permettant de traiter une question socialement vive (Pache, 2014). Plus récemment, deux équipes travaillant sur la base d'un dispositif Lesson Study (Lewis et Hurd, 2011) - une équipe en histoire et une équipe en géographie - ont mis en évidence différents modes de construction d'une communauté discursive scolaire à partir d'une triple perspective théorique : didactique, psychosociologique et langagière (Breithaupt, et al., 2017). Enfin, dans le monde germanophone, Handro et Schönnemann (2010) ont pu montrer que l'histoire et la langue sont inextricablement liées. Ces chercheur.e.s définissent non seulement un domaine de recherche émergent en didactique, mais ils insistent sur l'usage de la langue dans la discipline, et en particulier sur la construction des problèmes historiques. Il s'agirait aussi là d'un défi majeur pour l'enseignement.

De manière générale, le fait d'étudier la notion de pratiques langagières en contexte scolaire permet de prendre en compte, d'une part, la contextualisation liée à la situation et, d'autre part, tous les registres de pensée 
de l'individu. Ce sujet n'est ainsi pas seulement perçu comme sujet scolaire, mais aussi comme sujet social, puisque les productions langagières de l'élève sont alors vues comme une :

Production hétérogène dans laquelle se trouvent obligatoirement présentes les dimensions culturelles, sociales, langagières, tout à la fois singulières (propres au sujet qui les produit) et partagées (propres au groupe qui les reconnaît et en a élaboré les formes) et, de ce fait, normées. (Bautier, 2001, p. 127)

L'étude fine des interactions scolaires permet dès lors de mettre en évidence trois grandes tensions : la tension entre les dimensions préconstruites et émergentes; la tension entre les dimensions collectives et individuelles ; la tension entre les invariants et les spécificités des interactions scolaires (Nonnon, 2008). De tels travaux mettent à distance une conception strictement linguistique des pratiques langagières (Souplet, 2012), puisque ce n'est pas le degré de maîtrise linguistique qui a une incidence sur les apprentissages, « c'est dans la dimension discursive des productions que se réalisent les usages langagiers ", notamment ceux qui ont à voir avec les apprentissages (Bautier, 1995, p. 193).

C'est dans cet ensemble de problématiques que s'inscrit ce numéro thématique qui porte sur les pratiques langagières dans l'enseignement et dans la formation des enseignant.e.s. Plus particulièrement, ce dossier vise à interroger, d'un point de vue didactique, les formes ou les modalités opératoires pouvant recouvrir ces pratiques dans les contextes de la classe et de la formation (initiale ou continue) à l'enseignement. Il s'agit également d'identifier des processus qui seraient propres à certaines disciplines (ou domaines disciplinaires), ainsi que des phénomènes qui se retrouveraient dans toute situation d'enseignement, quelle que soit la discipline.

Les contributions présentées dans ce numéro thématique se répartissent ainsi en trois groupes : les pratiques langagières qui concernent principalement les disciplines des sciences de la nature et de la technologie (a), les pratiques langagières qui concernent les disciplines des sciences humaines et sociales (b) et les pratiques langagières qui renvoient à la littératie scolaire (c). Celles-ci sont de nature transversale et touchent ainsi toutes les disciplines.

Dans le premier ensemble de textes (a), Jaubert et Rebière se questionnent sur les outils linguistiques et discursifs qui permettent d'identifier la construction des langages disciplinaires scolaires, en prenant comme exemples l'enseignement des sciences de la nature et du français. Elles préconisent notamment de ne pas dissocier la forme et le fond afin de favoriser les apprentissages.

Bisault examine les enjeux et les problèmes liés à l'utilisation de la langue dans le contexte des activités scientifiques à l'école primaire. Il montre l'ambiguïté de certaines tâches langagières ainsi que l'imbrication des difficultés scientifiques et langagières.

Dans leur contribution, Kohler et Chabloz mettent en évidence la nécessité de coordonner les registres sémiotiques dans le cadre de l'enseignement de la physique. Ils insistent notamment sur l'importance de l'analyse $a$ priori afin de limiter les situations de malentendu.

La contribution de Roy propose une réflexion conceptuelle sur l'enseignement de la technologie. Six pratiques épistémiques d'ingénierie sont définies en adéquation avec les pratiques sociotechniques de référence.

Pour initier le deuxième ensemble de textes (b), Pache et Breithaupt analysent une leçon de géographie donnée successivement dans trois classes différentes, afin de mettre en évidence des pratiques langagières favorisant le processus de secondarisation. Une dissymétrie entre le travail cognitif des élèves et celui de l'enseignant·e est relevée.

Dans le cadre de l'histoire, Doussot étudie les conditions de transformation des pratiques langagières d'enquête à l'école primaire, et, notamment, l'enjeu central d'articulation des temporalités multiples.

Puis, le texte d'Handro et Kilimann poursuit la réflexion sur l'enseignement de l'histoire avec une perspective de "professionnalisation historico-didactique». En conclusion, les auteurs font l'hypothèse que cette approche centrée sur la langue n'est pas juste bénéfique pour les professeur.e.s d'histoire. Elle devrait également prendre place dans tous les autres domaines disciplinaires.

Dans le troisième ensemble de textes (c), le texte de Lange pose d'emblée une notion centrale : celle de "pratique linguistique éducative " qui est transversale aux disciplines. Sur la base d'une étude empirique, l'auteure insiste sur l'importance de la décontextualisation, afin de mobiliser une véritable compétence, et sur le rôle central de l'enseignant.e.

Bautier et Vinel interrogent ensuite la façon dont les pratiques de classe et la nature des échanges aident ou non les élèves à se familiariser avec des usages du langage à visée d'apprentissage. Leur contribution propose par ailleurs quelques pistes permettant de réduire les inégalités scolaires.

Enfin, la contribution de Schmellentin et Lindauer conclut le dossier en insistant sur le concept d' "enseignement d'une matière conscient du langage ». Ces auteurs proposent de travailler sur les points communs aux différentes disciplines scolaires, et donc sur la création de nouveaux concepts interdisciplinaires favorisant l'acculturation des élèves. 


\section{Références bibliographiques :}

Audigier, F. (2000). Histoire, géographie, éducation civique: trois disciplines aux prises avec la diversité narrative. Dans Y. Reuter et C. Tauveron (dir.), Repères, Diversité narrative (p. 121-143). INRP.

Audigier, F. (2007). De l'usage des récits pour dire l'histoire, la géographie, l'éducation citoyenne. Pratiques, 133-134, 77-99.

Audigier, F., Fink, N., Freudiger, N. et Haeberli, P. (dir.). (2011). L'éducation en vue du développement durable: sciences sociales et élèves en débats. Université de Genève.

Bachelard, G. (1993). La formation de l'esprit scientifique (3e éd.). Vrin.

Bachelard, G. (1998). Le rationalisme appliqué (3e éd.). PUF.

Bachelard, S. (1979). Quelques aspects historiques des notions de modèle et de justification des modèles. Dans P. Delattre et M. Thellier (dir.), Elaboration et justification des modèles (tome 1, p. 3-19). Maloine Éditions.

Bakthine, M. (1984). Esthétique de la création verbale. Gallimard.

Bautier, E. (1995). Pratiques langagières, pratiques sociales. De la sociolinguistique à la sociologie du langage. L'Harmattan.

Bautier, E. (2001). Pratiques langagières et scolarisation, Note de synthèse. Revue Française de Pédagogie, 137, 117-161.

Becker-Mrotzek, M., Schramm, K., Thürmann, E. et Vollmer, H.J. (dir.) (2013). Sprache im Fach. Sprachlichkeit und fachliches Lernen. Waxmann.

Bécu-Robinault, K. (2015). Un cadre épistemo-sémiotique pour concevoir des séances et analyser des pratiques d'étude et d'enseignement de la physique (Habilitation à diriger des recherches, Université Toulouse Jean-Jaurès, France).

Bigot, V. (2017). L'analyse des interactions didactiques dans la formation initiale des enseignants de français langue seconde. Communiquer, 17, 5-27.

Bisault, J. et Rebiffé, C. (2011). Découverte du monde et interactions langagières à l'école maternelle : construire ensemble un objet d'investigation scientifique. Carrefours de l'éducation, 3, 13-28. https://doi.org/10.3917/cdle.hs01.0013

Breithaupt, S., Ducrey Monnier, M., Pache, A. et Honoré, E. (2017). Formation à l'enseignement de la géographie et de l'histoire : les enjeux de la secondarisation dans un dispositif Lesson Study. Actes de la recherche de la HEP-BEJUNE, 11, 63-80.

Bucheton, D. et Dezutter, O. (2016). Le développement des gestes professionnels dans l'enseignement du français. De Boeck. https://doi.org/10.3917/dbu.buche.2008.01

Bunge, M. (1983). Épistémologie. Maloine.

Canguilhem, G. (1955). La formation du concept de réflexe aux XVIIe et XVIIIe siècles. PUF.

Canguilhem, G. (1988). Idéologie et rationalité dans l'histoire des sciences de la vie. Vrin.

Chevallard Y. (1991). Dimension instrumentale, dimension sémiotique de l'activité mathématique. Séminaire de Didactique des Mathématiques et de l'Informatique de Grenoble. Année 1990-1991, LSD2, IMAG, Université J. Fourier, 103-117.

Cummins, J. (1979). Cognitive/academic language proficiency, linguistic interdependence, the optimum age question and some other matters. Working Papers on Bilingualism, 19, 121-129.

Doussot, S. (2011). Didactique de l'histoire: outils et pratiques de l'enquête historienne en classe. Presses universitaires de Rennes. https://doi.org/10.4000/rfp.4046

Driver, R., Newton, P. et Osborne, J. (1998). Establishing the norm of scientific argumentation in classroom. Science Education, $84,1-35$.

Drouin, A. M. (1988). Le modèle en question. Aster, 7, 1-20.

Duval, R. (1996). Quel cognitif retenir en didactique des mathématiques ? Recherche en Didactique des Mathématiques, 16(3), 349-382.

El-Mekaoui, B. et Regnier, J.-C. (2016). La narration de recherche dans l'enseignement des mathématiques à l'école primaire. Colloque international « La Pédagogie et la Didactique des disciplines scientifiques, Tanger, Maroc.

Fadhlaoui, N. (2016). Le métalangage grammatical du français dans les classes tunisiennes: le cas de la notion de complément. Thèse de doctorat en linguistique. Université Paris Sorbonne.

Feilke, H. (2012). Bildungssprachliche Kompetenzen fördern und entwickeln. Praxis Deutsch, 39(233), 4-13.

Filliettaz, L. et Schubauer-Leoni, M.-L. (dir.), Processus interactionnels et situations éducatives. De Boeck. https://doi.org/10.3917/ dbu.filli.2008.01.0043

Fillon, P. et Peterfalvi, B. (2004). L'argumentation dans l'apprentissage scientifique au collège. Aster, 38 (Numéro thématique : Interactions langagières 2). https://doi.org/10.4267/2042/8835

Fürstenau, S. et Gomolla, M. (2009). Migration und schulischer Wandel : Elternbeteiligung. Verlag für Sozialwissenschaften.

Fürstenau, S. et Gomolla, M. (2011). Migration und schulischer Wandel : Mehrsprachig. Verlag für Sozialwissenschaften.

Fürstenau, S. et Gomolla, M. (2012). Migration und schulischer Wandel : Leistungsbeurteilung. Verlag für Sozialwissenschaften.

Gilbert, J. K. et Boulter, C. (2000). Developing Models Science Education. Kluwer.

Gogolin, I. (2010). Was ist Bildungssprache? Grundschulunterricht Deutsch, 4, 4-5.

Halbwachs, F. (1974). La pensée physique chez l'enfant et le savant. Delachaux et Niestlé.

Hasni, A. (2010). Modèles et modélisation en enseignement scientifique : quelques aspects prioritaires à considérer. Spectre, 40(1), $10-13$

Handro, S. et Schönemann, B. (dir.) (2010). Geschichte und Sprache. LIT Verlag.

Jaubert, M. et Rebière, M. (2000). Observer l'activité langagière des élèves en sciences. Aster, 31, 173-195. https://doi. org/10.4267/2042/8757

Jaubert, M. et Rebière, M. (2001). Pratiques de reformulation et construction de savoirs. Aster, 33, 81-110. https://doi. org/10.4267/2042/8777

Johsua, S. et Dupin, J.-J. (1989). Représentations et modélisations : le débat scientifique dans la classe. Peter Lang.

Lalagüe-Dulac, S. (2012). Le rôle du langage dans la construction de savoirs historiques sensibles et de compétences éthiques. Spirale, supplément électronique au nº 49 (19-32).

Latour, B. et Woolgar, S. (1988). La Vie de laboratoire. La production des faits scientifiques. La Découverte. 
Lewis, C. C. et Hurd, J. (2011). Lesson study step by step. How Teacher Learning Communities Improve Instruction. Heinemann. Lhoste, Y. (2008). Problématisation, activités langagières et apprentissages dans les sciences de la vie. Étude de débats scientifiques dans la classe dans deux domaines biologiques : nutrition et évolution, thèse de doctorat, Université de Nantes.

Moscovici, S. et Hewstone, M. (1984). De la science au sens commun. Dans S. Moscovici (dir.), Psychologie sociale et problèmes sociaux (p. 538-566). PUF.

Nonnon, E. (2008). Tension et dynamiques des interactions dans les échanges scolaires. Dans L. Filliettaz, M.-L. Schubauer-Leoni (dir.), Processus interactionnels et situations éducatives (p. 43-65). De Boeck. https://doi.org/10.3917/dbu.filli.2008.01.0043

Orange, C. (1997). Problèmes et modélisation en biologie. Quels apprentissages pour le lycée ? PUF.

Orange, C. (2003). Débat scientifique dans la classe, problématisation et argumentation : le cas d'un débat sur la nutrition au cours moyen. Aster, 37, 83-107. https://doi.org/10.4267/2042/8821

Osborne, J., Erduran, S., Simon, S. et Monk, M. (2001). Enhancing the quality of argument in school science, School Science Review, 82(301), 63-70. https://doi.org/10.1002/tea.20035

Pache, A. (2014). L'alimentation à l'école. Enseigner une géographie renouvelée. Presses universitaires de Rennes.

Popper, K. (1991). La connaissance objective. Aubier.

Robardet, G. et Guillaud, J.-C. (1997). Éléments de didactique des sciences physiques. Presses Universitaires de France.

Schneeberger, P. (dir.) (2003). Argumentations et démonstrations dans les débats et discussions dans les classes. Rapport de recherche associative INRP - IUFM d'Aquitaine (coordination nationale J. Colomb).

Schneeberger, P. et Ponce, C. (2003). Tirer parti des échanges langagiers entre pairs pour construire des apprentissages en sciences. Aster, 37 (Numéro thématique : Interactions langagières 1). https://doi.org/10.4267/2042/8820

Schneeberger, P., Robisson, P., Liger-Martin, J. et Darley, B. (2007). Conduire un débat pour faire construire des connaissances en sciences. Aster, 45, 39-64. https://doi.org/10.4267/2042/16817

Souplet, C. (2012). Pratiques langagières et situation scolaire d'apprentissage : à propos d'un rapport complexe et résistant. Questions méthodologiques. Education et didactique, 1(6), 97-115. https://doi.org/10.4000/educationdidactique.1310

Tiberghien, A. (1994). Modeling as a basis for analyzing teaching-learning situations. Learning and instruction, 4(1), 71-87.

Walliser, B. (1977). Système et modèles. Seuil.

Vygotski, L. (1985/2013). Pensée et langage (4éd.). La Dispute. 